\title{
Expression of Clara cell 10-kDa protein and trefoil factor family 1 in patients with chronic rhinosinusitis and nasal polyps
}

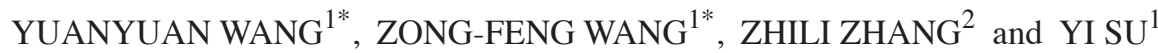 \\ Departments of ${ }^{1}$ Otolaryngology, Head and Neck Surgery and ${ }^{2}$ Orthodontics, \\ Dongying People's Hospital, Dongying, Shandong 257091, P.R. China
}

Received July 21, 2016; Accepted July 20, 2017

DOI: 10.3892/etm.2018.5725

\begin{abstract}
The current study measured the expression of Clara cell 10-kDa protein (CC10) and trefoil factor family 1 (TFF1) in the sinus mucosa of patients exhibiting chronic rhinosinusitis (CRS) and nasal polyps (NP). CC10 and TFF1 expression in the sinus mucosa of the control group and patients with CRS and NP was determined using reverse transcription-quantitative polymerase chain reaction (RT-qPCR), western blotting and immunohistochemistry. The correlation between CC10 and TFF1 expression was further analyzed using Spearman's correlation analysis. The expression of TFF1 was significantly increased in the sinus mucosa of patients with CRS and NP, whereas CC10 expression was significantly decreased compared with controls. Spearman's correlation analysis identified a negative correlation between CC10 and TFF1 expression in the sinus mucosa of patients with CRS and NP. The results of immunohistochemistry and RT-qPCR were consistent with each other. Hematoxylin and eosin staining revealed notable lesions in the mucous membranes, goblet cells and cilia of sinus mucosa samples from patients with CRS and NP. The negative correlation between $\mathrm{CC} 10$ and TFF1 expression during the progression of CRS and NP suggest that CC10 and TFF1 may serve important roles in its pathogenesis.
\end{abstract}

\section{Introduction}

Chronic rhinosinusitis (CRS) is one of the most common otorhinolaryngological diseases, which severely impairs quality of life and induces a heavy economic burden on patients (1). However, the etiology and pathogenesis of CRS remain unknown, making its diagnosis, classification and

Correspondence to: Dr Zong-Feng Wang, Department of Otolaryngology, Head and Neck Surgery, Dongying People's Hospital, 317 Nanyi Road, Dongying, Shandong 257091, P.R. China E-mail: prof_wang2016@163.com

*Contributed equally

Key words: chronic rhinosinusitis, nasal polyps, clara cell 10-kDa protein, trefoil factor family 1 treatment challenging (2). A greater understanding of the molecular pathological mechanisms underlying the onset of CRS may facilitate the identification of a novel method of diagnosis and the development of novel therapeutic strategies to treat the condition.

The majority of patients with CRS also experience nasal polyps (NP), which has a high rate of recurrence even following the administration of appropriate drugs and surgical treatments (3). This has led to the classification of CRS as either CRS with nasal polyps (CRSwNP) or CRS without nasal polyps (CRSsNP) (4). It has been demonstrated that the pro-inflammatory cytokine interleukin-32, is differentially expressed in the nasal epithelial cells of patients with CRSwNP and those with CRSsNP (5). Although the differential expression of inflammatory mediators in CRSwNP and CRSsNP has been previously demonstrated (6), further studies are required to provide a basis for the accurate diagnosis and the development of effective treatment strategies.

Clara cell 10-kDa protein (CC10), also known as uteroglobin, is a steroid-inducible member of the secretoglobin family that serves an important role in the regulation of anti-inflammatory and immunomodulatory activities $(7,8)$. CC10 is constitutively expressed in the epithelial cells of organs that directly communicate with the external environment, including the nose, bronchi and lungs (7-9). Furthermore, CC10 is an important mediator of inflammatory and allergic responses, as well as responses to malignant tumors of the respiratory system (10). Pulmonary CC10 protein serves a role in the T-cell-mediated inflammatory response by modulating expression of the T helper 2 cytokine (11). Furthermore, the histamine $\mathrm{H} 1$ receptor antagonist fexofenadine hydrochloride significantly elevates the expression of $\mathrm{CC} 10$ protein in nasal epithelial cells, which may partially account for the therapeutic effect of fexofenadine hydrochloride in the treatment of allergic disorders (12). Additionally, it has been demonstrated that increased levels of CC10 are associated with improvements in bronchial dysplasia and sputum cytometric assessment in patients at high risk of developing lung cancer (13). However, the expression of $\mathrm{CC10}$ in patients with CRS and NP and its role in the development of the disease, remain unknown.

The trefoil factor (TFF) family members TFF1, TFF2 and TFF3 are secreted from a variety of mucous epithelial tissues and function as important regulators of cell migration, immune reaction, mucosa repair, angiogenesis and 
tumorigenesis (14-16). TFF peptides have also been identified in many other organs, including the respiratory tract, gallbladder, prostate, uterus, thyroid gland, salivary glands, mammary glands and the nervous system (17). TFF1, also known as pS2, contains 60 amino acid residues and is mainly expressed in the gastric mucosa as a protective factor against gastric damage (18). However, it has also been demonstrated that TFF1 is widely expressed in human and mouse nasal mucosa, suggesting that it may also regulate the inflammatory response in the respiratory tract (19-21). The expression and function of TFF1 in CRS and NP remains unclear.

The present study investigated the differential expression of CC10 and TFF1 in the nasal mucosa of patients with CRS and NP to provide further information regarding the pathology of CRS.

\section{Materials and methods}

Patients and samples. Nasal mucosa samples were obtained from different patient groups as follows: i) 20 samples from patients who were diagnosed with CRS without NP and had undergone endoscopic sinus surgery for CRS at Dongying People's Hospital (Donying, China) (13 males, 7 females; age range, 22-70 years); ii) 20 samples from patients who were diagnosed with NP without CRS and had undergone endoscopic sinus surgery for NP at Dongying People's Hospital (14 males, 6 females; age range, 20-73 years); and iii) 18 samples from healthy controls (10 males, 8 females; age range, 18-55 years). All patients were recruited from September 2014 to October 2015 . All samples were stored at $-80^{\circ} \mathrm{C}$ immediately after collection. CRS and NP were diagnosed according to the combined criteria developed by the American Academy of Allergy, Asthma \& Immunology and American Academy of Otolaryngology-Head and Neck Surgery $(22,23)$. Diagnosis of CRS was based on typical sinusitis symptoms, including thick mucus, a blocked nose and pain in the face lasting $>12$ weeks and positive findings from a computer topography (CT) scan of the unilateral or bilateral nose. NP was determined by the presence of polyps in the middle meatus or nasal cavity detected by endoscopy or surgery. Normal nasal mucosa samples collected from the inferior turbinate or uncinate process of 18 patients with nasal septum deviation and no sign of anterior ethmoid sinusitis in CT and nasal endoscopy were used as healthy controls (Table I). These patients were also recruited from Dongying People's Hospital. The current study was approved by the Ethics Committee of Dongying People's Hospital and informed consent was obtained from all participants.

Reverse transcription-quantitative polymerase chain reaction $(R T-q P C R)$. Total RNA samples were extracted using the TRIzol ${ }^{\circledR}$ reagent (cat. no. 15596026; Invitrogen; Thermo Fisher Scientific, Inc., Waltham, MA, USA) according to the manufacturer's protocol. The ultraviolent absorbance of $2 \mu 1 \mathrm{RNA}$ diluted with $198 \mu 1$ Milli-Q water was measured at wavelengths of 260 and $280 \mathrm{~nm}$. The concentration and purity of RNA samples was calculated as follows: RNA concentration $(\mu \mathrm{g} / \mu \mathrm{l})=$ optical density $(O D)_{260} \times 4$ and RNA purity $=\mathrm{OD}_{260} / \mathrm{OD}_{280}$. RNA samples with a purity of $\mathrm{OD}_{260} / \mathrm{OD}_{280}>1.8$, were used for RT-qPCR. The cDNA was synthesized using a PrimeScript ${ }^{\mathrm{TM}}$ First Strand cDNA Synthesis kit (cat. no. 6110A; Takara Bio,
Table I. Clinical data of patients with CRS and NP.

\begin{tabular}{lccc}
\hline Feature & Control & CRS & NP \\
\hline Number of patients & 18 & 20 & 20 \\
Sex (male/female) & $10 / 8$ & $13 / 7$ & $14 / 6$ \\
Age (years) & $18-55$ & $22-70$ & $20-73$ \\
Skin Prick Test & 0 & 4 & 3 \\
History of asthma & 0 & 0 & 2 \\
History of smoking & 5 & 9 & 8 \\
Aspirin intolerance & 0 & 0 & 0 \\
\hline
\end{tabular}

CRS, chronic rhinosinusitis; NP, nasal polyps .

Inc., Otsu, Japan) according to the manufacturer's protocol. Reverse transcription was performed at $42^{\circ} \mathrm{C}$ for $1 \mathrm{~h}$ followed by RNA transcriptase inactivation at $72^{\circ} \mathrm{C}$ for $10 \mathrm{~min}$. qPCR reactions were subsequently performed using a SYBR Fast qPCR mix (cat. no. RR430A; Takara Bio, Inc.). The primer sequences used in qPCR are presented in Table II. qPCR was performed using the following settings: Pre-denaturation at $95^{\circ} \mathrm{C}$ for $10 \mathrm{sec}$, followed by 40 cycles of denaturation at $95^{\circ} \mathrm{C}$ for $5 \mathrm{sec}$ and elongation at $60^{\circ} \mathrm{C}$ for $30 \mathrm{sec}$. The threshold cycle of each sample was identified using LightCycler version 96 software combined with the LightCycler 96 Real-Time PCR system (both Roche Applied Science, Penzberg, Germany). Relative expression was calculated using the $2^{-\Delta \Delta \mathrm{Cq}}$ method and $\beta$-actin was used as the internal standard. Expression of $\mathrm{CC} 10$ and TFF1 in patients with CRS and NP were compared with that in normal nasal mucosa of the inferior turbinate collected from the control group.

Immunohistochemical analysis and hematoxylin and eosin $(H \& E)$ staining. The expression of TFF1 and CC10 was determined using immunohistochemistry, following the streptavidin biotin-peroxidase complex (SABC) method. Tissues were fixed overnight in $4 \%$ paraformaldehyde at room temperature, embedded in paraffin and sliced into $4-\mu \mathrm{m}$-thick serial sections. Slides were deparafinized in two treatments of xylene (each $5 \mathrm{~min}$ ) and transferred to $100 \%$ alcohol for two treatments (each $3 \mathrm{~min}$ ), then once through 95, 70 and 50\% alcohols respectively for $3 \mathrm{~min}$ each. The slides were then incubated in $3 \%$ hydrogen peroxide to remove endogenous oxidases for $10 \mathrm{~min}$ at room temperature. After rinsing twice in PBS (5 min each), a citrate buffer were used to performed antigen retrieval $\left(95-100^{\circ} \mathrm{C}\right.$ for $\left.10 \mathrm{~min}\right)$. Normal $5 \%$ goat serum (cat. no. 5425, Cell Signaling Technology, Inc., Danvers, MA, USA) was used as a blocking reagent and the slides were incubated for $1 \mathrm{~h}$ at a room temperature. The remaining steps were performed using an SABC kit purchased from Wuhan Boster Biological Technology Ltd. (Wuhan, China) and a diaminobenzidine kit (cat. no. ZLI-9017), which was purchased from Beijing ZSGB-Bio Co., Ltd. (Beijing, China). Each kit was used according to the manufacturer's protocol. PBS buffer was applied to replace primary antibodies in the negative control. Rabbit anti-human CC10 (cat. no. sc-25555) and mouse anti-human TFF1 (cat. no. sc-271464) polyclonal antibodies were purchased from Santa Cruz Biotechnology, 
Table II. Primer sequences used in reverse transcription-quantitative polymerase chain reaction.

Primer sequences $\left(5^{\prime}-3^{\prime}\right)$

\begin{tabular}{lll}
\cline { 2 - 3 } Gene & \multicolumn{1}{c}{ Forward } & \multicolumn{1}{c}{ Reverse } \\
\hline CC10 & GATCAAGACATGAGGGAGGCA & CACAGTGAGCTTTGGGCTATTT \\
TFF1 & CAATGGCCACCATGGAGAAC & AACGGTGTCGTCGAAACAGC \\
\hline
\end{tabular}

TFF1, trefoil factor family 1; CC10, clara cell 10-kDa protein.

Inc. (Dallas, TX, USA) and used at dilutions of 1:800 and 1:100, respectively. Slides were incubated with CC10 and TFF1 antibodies for $1 \mathrm{~h}$ at room temperature. Positive staining was defined by fine yellow particles in the field of view using high-magnification optical microscopy. H\&E staining was conducted following a previously published protocol (24).

Western blot analysis. Total protein from human nasal mucosa tissues was extracted using radioimmunoprecipitation assay lysis buffer (Beyotime Institute of Biotechnology, Haimen, China), following the manufacturer's protocol. Following the measurement of protein concentration using a Pierce ${ }^{\mathrm{TM}}$ BCA Protein assay kit (no. 23227; Pierce; Thermo Fisher Scientific, Inc.), total protein from the control, CRS and NP groups $(15 \mu \mathrm{g})$ was subjected to $15 \%$ SDS-PAGE and blotted onto PVDF membranes. PVDF membranes were then blocked with TBS buffer containing $5 \%$ milk powder for $2 \mathrm{~h}$ at room temperature, washed 3 times for 5 min using TBS buffer, incubated with anti-CC10, anti-TFF1 or anti-GAPDH $(1: 1,000$; cat no. AF0006; Beyotime Institute of Biotechnology) antibodies for $1 \mathrm{~h}$ at room temperature, washed three times for $5 \mathrm{~min}$ using TBS buffer and incubated with horseradish peroxidase-conjugated secondary antibodies (1:10,000; cat nos. A4416 and A6154; Sigma-Aldrich; Merck KGaA, Darmstadt, Germany) for $1 \mathrm{~h}$ at room temperature. Rabbit anti-human CC10 (1:500; cat. no. sc-25555) polyclonal antibodies and mouse anti-human TFF1 (1:500, cat. no. sc-271464) polyclonal antibodies were purchased from Santa Cruz Biotechnology, Inc. The membranes were subsequently rinsed with TBS three times, the blots were visualized using an enhanced chemiluminescence western blot detection reagent (cat. no. 321096; Thermo Fisher Scientific, Inc.) and exposed to X-ray film.

Statistical analysis. Data analysis was performed using SPSS version 16.0 (SPSS, Inc., Chicago, IL, USA) and $\mathrm{P}<0.05$ was determined to indicate a statistically significant difference. One-way analysis of variance was used followed by Tukey's post-hoc test. The correlation between TFF1 and CC10 expression was analyzed using Spearman's correlation analysis.

\section{Results}

Expression of CC10 in human nasal mucosa from patients with CRS and NP. The expression of CC10 mRNA in the control, CRS and NP groups was initially analyzed using
RT-qPCR. The expression of CC10 mRNA in the CRS group was significantly decreased compared with that of the control group $(\mathrm{P}<0.05$; Fig. 1). Furthermore, the expression of $\mathrm{CC} 10$ mRNA was significantly decreased in the NP group compared with that of the CRS group ( $\mathrm{P}<0.05$; Fig. 1$)$.

Expression of TFF1 in the nasal mucosa of patients with CRS and NP. The potential role served by TFF1 in the pathogenesis of CRS and NP was investigated by determining the expression of TFF1 in the control, CRS and NP groups using RT-qPCR. The results demonstrated that the expression of TFF1 in the CRS group was significantly increased compared with that in the control group ( $\mathrm{P}<0.05$; Fig. 2$)$. TFF1 expression in the NP group exhibited a further significant increase compared with the CRS group ( $\mathrm{P}<0.05$; Fig. 2$)$.

CC10 and TFF1 protein expression in human nasal mucosa of patients with CRS and NP. The expression of CC10 and TFF1 proteins in nasal mucosa tissues from the control, CRS and NP groups was confirmed using western blot analysis. Two samples of HC, CRS and NPS were randomly selected to perform western blot analysis on. The expression of $\mathrm{CC} 10$ protein in the CRS and NP groups was lower compared with those of the control group and a larger decrease in the expression of CC10 protein was observed in the NP group compared with the CRS group (Fig. 3). This is consistent with the results of the RT-qPCR analysis (Fig. 2). By contrast, the expression of TFF1 protein in the CRS and NP groups was higher compared with the control (Fig. 3). There was no difference in expression of TFF1 protein between the CRS and NP groups (Fig. 3).

Immunohistochemical analysis. Immunohistochemical staining was performed using the SABC method to measure the expression of TFF1 and CC10. The results demonstrated that TFF1 was primarily expressed in goblet and ciliated cells and distributed in the cytoplasmic regions surrounding the nucleus (Fig. 4A-C). The expression of TFF1 in the mucosa tissues of patients in the CRS (Fig. 4B) and NP (Fig. 4C) groups was increased compared with that of the control group (Fig. 4A). Additionally, the results determined that CC10 was mainly expressed in the epithelial cells of the nasal mucosa from all groups (Fig. 4D-F). The staining densities in the control group (Fig. 4D) were lower compared with the CRS (Fig. 4E) and NP (Fig. 4F) groups, which clearly demonstrated that expression of $\mathrm{CC} 10$ is decreased in patients with CRS and NP. 


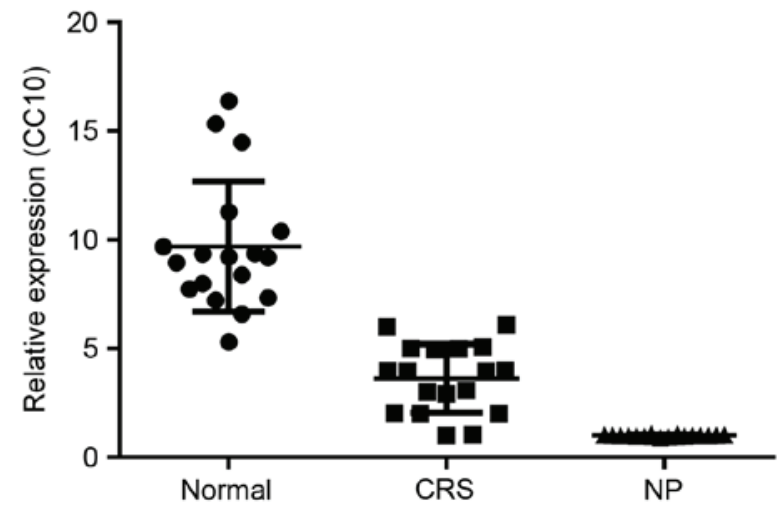

Figure 1. CC10 mRNA expression in the nasal mucosa of patients with CRS and NP. The relative expression of CC10 mRNA was analyzed using reverse transcription-quantitative polymerase chain reaction. $\beta$-actin was used as the internal standard. CRS, chronic rhinosinusitis; NP, nasal polyps; CC10, clara cell 10-kDa protein.

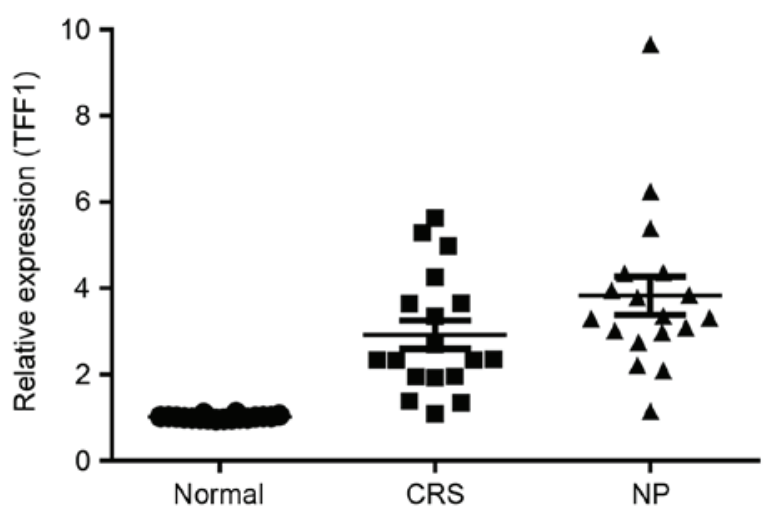

Figure 2. TFF1 mRNA expression in the nasal mucosa of patients with CRS and NP. The relative expression of TFF1 mRNA was analyzed using reverse transcription-quantitative polymerase chain reaction. $\beta$-actin was used as the internal standard. CRS, chronic rhinosinusitis; NP, nasal polyps; TFF1, trefoil factor family 1 .

The results of H\&E staining clearly identified a lesion in the CRS and NP groups, which was not present in the control group (Fig. 4G-I). A thickened mucous layer, hyperplastic goblet cells and deciduous cilia were also detected in the CRS (Fig. 4H) and NP (Fig. 4I) groups however, these were more marked in the NP group compared with the CRS group.

Correlation of CC10 and TFF1 expression in human nasal mucosa of patients with CRS and NP. Considering the differential expression of CC10 and TFF1 in human nasal mucosa from patients with CRS and NP, a Spearman's correlation analysis was performed. The results demonstrated that there was a significant negative correlation between the expression of CC10 and TFF1 in the tissues of patients with CRS and NP ( $r=-0.89, \mathrm{P}<0.05$; Fig. 5), suggesting that $\mathrm{CC} 10$ and TFF1 may interact during the development of CRS and NP.

\section{Discussion}

The pathogenesis of CRS and NP is not fully understood; however, previous studies have focused on several hypotheses, including bacterial infections, fungal colonization, local

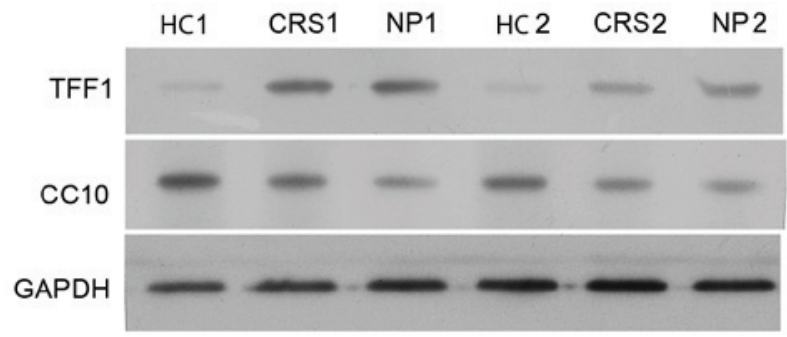

Figure 3. The expression of CC10 and TFF1 protein in the nasal mucosa of patients with CRS and NP, as well as HCs. Two random samples of CRS, $\mathrm{NP}$ and $\mathrm{HC}$ were selected and the expression of CC10 and TFF1 protein was determined by western blot analysis, using antibodies against CC10 and TFF1. CRS, chronic rhinosinusitis; NP, nasal polyps; TFF1, trefoil factor family 1 ; CC10, clara cell 10-kDa protein; $\mathrm{HC}$, healthy control.

anatomical structure variation, trauma, allergic reactions and environmental pollution. CRS and NP may be caused by these factors and mediated by mucosal immune dysfunction and excessive inflammatory reactions. These may therefore serve a critical role in the early development of CRS and NP and indicates that treating local mucosal immune disorders and excessive inflammatory reaction may attenuate the development of CRS and NP $(25,26)$.

The role served by $\mathrm{CC} 10$ in the progression and development of CRS and NP remains controversial. It has been demonstrated that CC10 is expressed in the goblet, non-mucinous and non-ciliated cells of the nasal sinus mucosa epithelium and that the number of CC10-positive cells is negatively correlated with inflammatory cell infiltration and the number of goblet cells. This indicates that downregulation of CC10 expression may lead to anti-inflammatory network dysfunction in the epithelial cells of the upper respiratory tract and a persistent severe inflammatory response eventually leads to the formation of NP (9). CC10 expression is negatively correlated with preoperative CT scores, as well as postoperative nasal endoscopy and symptom scores (8). By contrast, fexofenadine hydrochloride elevates the expression of CC10 protein in nasal epithelial cells, which may partially account for its therapeutic effect on allergic disorders (12). Furthermore, increased CC10 expression is associated with improvements in bronchial dysplasia and sputum cytometric assessment in patients at high risk of developing lung cancer (13). A previous study focusing on the effect of inflammatory cytokines on $\mathrm{CC} 10$ expression using nasal mucosa tissue culture identified that tumor necrosis factor- $\alpha$, interleukin (IL)- $1 \beta$ and IL-4 inhibited the expression of CC10, whereas IL-10 and interferon- $\gamma$ promoted the expression of $\mathrm{CC10}$ (8). These contradictory results suggest that $\mathrm{CC10}$ expression is very sensitive to pathological conditions and its differential expression may be context-dependent. In the present study, CC10 expression was significantly increased in the nasal mucosa tissues of patients with CRS and NP compared with the control group, which further demonstrates that CC10 serves a role in the pathology of CRS and NP.

Previous studies have demonstrated that TFF peptides are highly expressed in the goblet cells, submucosal glands and ciliary epithelial cells of the respiratory tract; however, TFF has not been identified in the alveolar epithelium. Among the three TFF peptides, TFF3 exhibited the highest expression in the human respiratory tract, followed by TFF1, whereas TFF2 
A

B

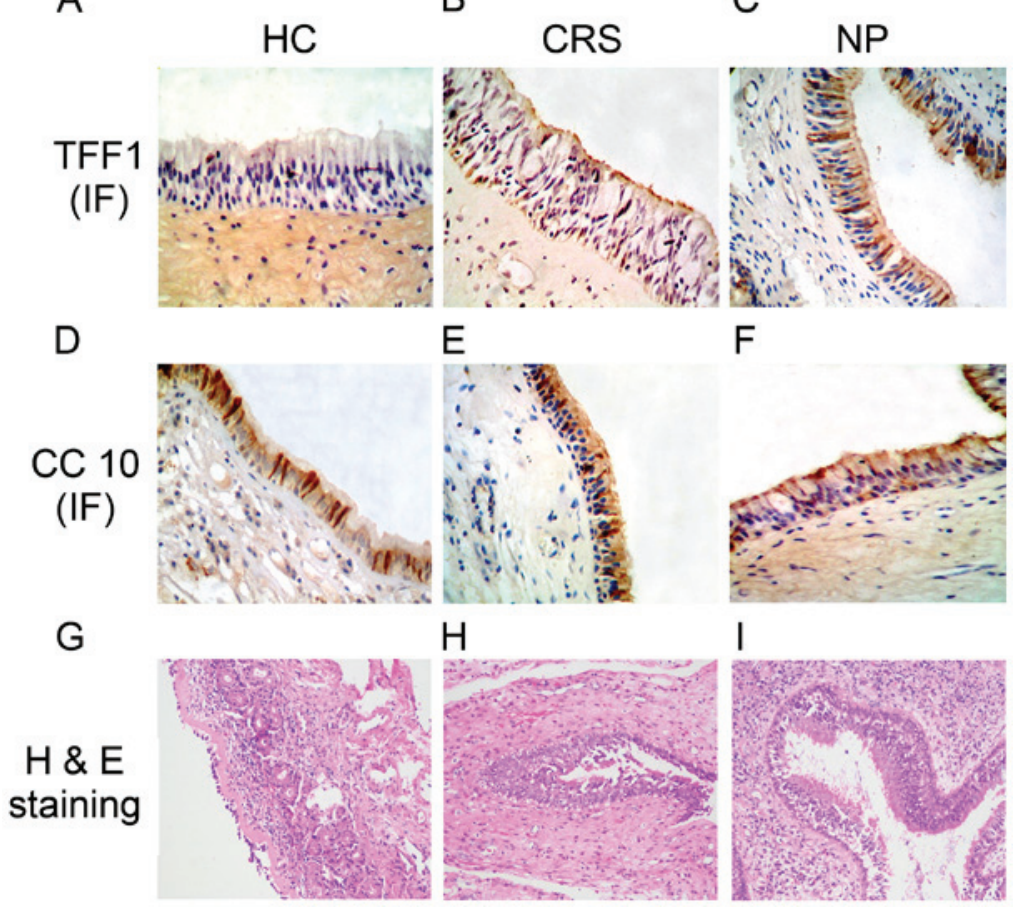

Figure 4. Immunohistochemistry and H\&E staining of CC10 and TFF1 expression in the nasal mucosa of patients with CRS and NP. (A) TFF1 expression in the HC group. (B) TFF1 expression in the CRS group. (C) TFF1 expression in the NP group. (D) CC10 expression in the HC group. (E) CC10 expression in the CRS group. (F) CC10 expression in the NP group. Brown particles indicate CC10 or TFF1-positive staining. (G) H\&E staining in the HC group. (H) H\&E staining in the CRS group. (I) H\&E staining in the NP group. Magnification, x400. HC, healthy control; CRS, chronic rhinosinusitis; NP, nasal polyps; TFF1, trefoil factor family 1; CC10, clara cell 10-kDa protein; H\&E, hemotoxylin and eosin.

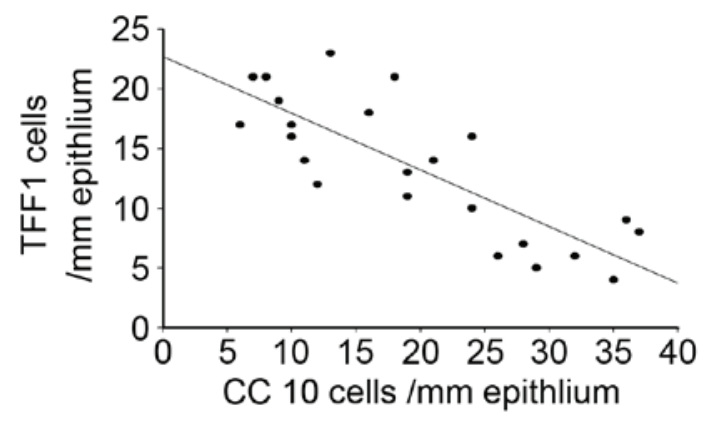

Figure 5. Correlation between CC10 and TFF1 expression in the nasal mucosa of patients with CRS and NP. The numbers of CC10- and TFF1-positive cells were counted and a negative correlation between CC10 and TFF1 expression was determined by Spearman correlation analysis using SPSS 16.0 software. $\mathrm{r}=-0.89 ; \mathrm{P}<0.05$. CRS, chronic rhinosinusitis; NP, nasal polyps; TFF1, trefoil factor family 1; CC10, clara cell 10-kDa protein.

was undetectable $(19,20,27)$. The current study analyzed the expression of TFF1 in human nasal mucosa using RT-qPCR and identified a significant decrease in the expression of TFF1 in the nasal mucosa of patients with CRS and NP. Immunohistochemistry and western blot analysis also clearly demonstrated that the expression of TFF1 protein was significantly increased in patients with CRS and NP, indicating that TFF1 may serve an important role in the progression of the disease. It has been demonstrated that TFF1 is mainly expressed in the goblet cells, cilia epithelial cells and mucosal glands of the respiratory tract from the nasal cavity to the bronchioles and its expression pattern overlaps with the secretion of airway mucin, which is distributed in the cytoplasmic regions surrounding the nucleus (28). In combination with the results of the current study, the expression of TFF in the human nasal mucosa tissues suggests that they serve a potential role in the pathology of CRS and NP.

Furthermore, it was previously demonstrated in that there was a negative correlation between the expression of $\mathrm{CC} 10$ and TFF1 during the progression of inflammation and alteration of the cell phenotype (29). However, the association between CC10 and TFF1 expression in human nasal mucosa tissues during the development CRS and NP remains unknown. The current study demonstrated that the expression of $\mathrm{CC} 10$ was significantly increased in the nasal mucosa tissues of patients with CRS and NP, whereas the expression of TFF1 was significantly decreased. Spearman's correlation analysis identified a negative correlation between the expression of TFF1 and CC10.

TFF1 is an important regulator of epithelial cell migration and the inflammatory process in the airway, which may serve an important regulatory role in the process of airway defense and injury repair $(15,20)$. TFF1 delays the transition from $\mathrm{G} 1$ to the S-phase in the cell cycle, thus regulating tumor inhibition, mucosal protection, cell apoptosis and differentiation (30). The expression of TFF1 in inflammation and malignant tumors indicates the status of prognosis and is significantly negatively correlated with the therapeutic effect of hormone therapy $(29,31)$. The decreased expression of TFF1 and its negative correlation with $\mathrm{CC} 10$ in human nasal mucosa in the current study suggests that TFF1 deficiency may contribute to the onset and progression of CRS and NP, and regulation of the expression of TFF1 and CC10 may be a potential treatment strategy for patients with CRS and NP. 
In conclusion, the current study identified the differential expression of CC10 and TFF1 in human nasal mucosa tissues from patients with CRS and NP. The negative correlation between $\mathrm{CC} 10$ and TFF1 suggests that they may serve a role the pathogenesis of CRS and NP. Therefore, the expression of these two markers may be a potential target for the diagnosis and treatment of CRS and NP in the future.

\section{References}

1. Fokkens W, Lund V and Mullol J; European Position Paper on Rhinosinusitis and Nasal Polyps group: European position paper on rhinosinusitis and nasal polyps 2007. Rhinol Suppl 20: 1-136, 2007.

2. Rosenfeld RM, Andes D, Bhattacharyya N, Cheung D, Eisenberg S, Ganiats TG, Gelzer A, Hamilos D and Hudgins PA III: Clinical practice guideline: Adult sinusitis. Otolaryngol Head Neck Surg 137 (3 Suppl): S1-S31, 2007.

3. Kaliner MA, Osguthorpe JD, Fireman P, Anon J, Georgitis J, Davis ML, Naclerio R and Kennedy D: Sinusitis: Bench to bedside. Current findings, future directions. J Allergy Clin Immunol 99 (Suppl): S829-S848, 1997.

4. Polzehl D, Moeller P, Riechelmann H and Perner S: Distinct features of chronic rhinosinusitis with and without nasal polyps. Allergy 61: 1275-1279, 2006.

5. Keswani A, Chustz RT, Suh L, Carter R, Peters AT, Tan BK, Chra R, Azam T and Dinarello CA: Differential expression of interleukin-32 in chronic rhinosinusitis with and without nasal polyps. Allergy 67: 25-32, 2011.

6. Van Zele T, Claeys S, Gevaert P, Van Maele G, Holtappels G, Van Cauwenberge P and Bachert C: Differentiation of chronic sinus diseases by measurement of inflammatory mediators. Allergy 61 : 1280-1289, 2006.

7. Singh G and Katyal SL: Clara cell proteins. Ann N Y Acad Sci 923: 43-58, 2000.

8. Liu Z, Lu X, Zhang XH, Bochner BS, Long XB, Zhang F, Wang $\mathrm{H}$ and Cui $\mathrm{YH}$ : Clara cell $10-\mathrm{kDa}$ protein expression in chronic rhinosinusitis and its cytokine-driven regulation in sinonasal mucosa. Allergy 64: 149-157, 2009.

9. Liu Z, Kim J, Sypek JP, Wang IM, Horton H, Oppenheim FG and Bochner BS: Gene expression profiles in human nasal polyp tissues studied by means of DNA microarray. J Allergy Clin Immunol 114: 783-790, 2004

10. Liu Y, Yu HJ, Wang N, Zhang YN, Huang SK, Cui YH and Liu Z: Clara cell $10-\mathrm{kDa}$ protein inhibits $\mathrm{T}(\mathrm{H}) 17$ responses through modulating dendritic cells in the setting of allergic rhinitis. J Allergy Clin Immunol 131: 387-394.e1-12, 2013.

11. Hung $\mathrm{CH}$, Chen LC, Zhang Z, Chowdhury B, Lee WL, Plunkett B, Chen CH, Myers AC and Huang SK: Regulation of TH2 responses by the pulmonary Clara cell secretory $10-\mathrm{kd}$ protein. J Allergy Clin Immunol 114: 664-670, 2004.

12. Nogaki T, Asano K, Furuta A, Kanai K, Suzaki I, Kanei A and Suzaki H: Enhancement of clara cell $10-\mathrm{kD}$ protein (CC10) production from nasal epithelial cells by fexofenadine hydrochloride. Asian Pac J Allergy Immunol 30: 139-145, 2012.

13. Chen J, Lam S, Pilon A, McWilliams A, Macaulay C and Szabo E: Higher levels of the anti-inflammatory protein CC10 are associated with improvement in bronchial dysplasia and sputum cytometric assessment in individuals at high risk for lung cancer. Clin Cancer Res 14: 1590-1597, 2008.
14. Oertel M, Graness A, Thim L, Bühling F, Kalbacher H and Hoffmann W: Trefoil factor family-peptides promote migration of human bronchial epithelial cells: Synergistic effect with epidermal growth factor. Am J Respir Cell Mol Biol 25: 418-424, 2001.

15. Hoffmann W: Trefoil factors TFF (trefoil factor family) peptide-triggered signals promoting mucosal restitution. Cell Mol Life Sci 62: 2932-2938, 2005

16. Kjellev S: The trefoil factor family-small peptides with multiple functionalities. Cell Mol Life Sci 66: 1350-1369, 2009.

17. Belovari T, Bijelić N, Tolušić Levak M and Baus Lončar $\mathrm{M}$ Trefoil factor family peptides TFF1 and TFF3 in the nervous tissues of developing mouse embryo. Bosn J Basic Med Sci 15: 33-37, 2015.

18. Thim L and May FE: Structure of mammalian trefoil factors and functional insights. Cell Mol Life Sci 62: 2956-2973, 2005.

19. Lee SH, Lee SH, Oh BH, Lee HM, Choi JO and Jung KY: Expression of mRNA of trefoil factor peptides in human nasal mucosa. Acta Otolaryngol 121: 849-853, 2001.

20. dos Santos Silva E, Ulrich M, Döring G, Botzenhart K and Gött P: Trefoil factor family domain peptides in the human respiratory tract. J Pathol 190: 133-142, 2000.

21. Kouznetsova I, Chwieralski CE, Bälder R, Hinz M, Braun A, Krug $\mathrm{N}$ and Hoffmann W: Induced trefoil factor family 1 expression by trans-differentiating Clara cells in a murine asthma model. Am J Respir Cell Mol Biol 36: 286-295, 2007.

22. Slavin RG, Spector SL, Bernstein IL, Kaliner MA, Kennedy DW, Virant FS, Wald ER, Khan DA, Blessing-Moore J, Lang DM, et al: The diagnosis and management of sinusitis: A practice parameter update. J Allergy Clin Immunol 116 (6 Suppl): S13-S47, 2005.

23. Scadding GK, Durham SR, Mirakian R, Jones NS, Drake-Lee AB, Ryan D, Dixon TA, Huber PA and Nasser SM; British society for allergy and clinical immunology: BSACI guidelines for the management of rhinosinusitis and nasal polyposis. Clin Exp Allergy 38: 260-275, 2008.

24. Zou Y, Wang Y, Wang SB, Kong YG, Xu YU, Tao ZZ and Chen SM: Characteristic expression and significance of CCL19 in different tissue types in chronic rhinosinusitis. Exp Ther Med 11: 140-146, 2016.

25. Norlander T, Westrin KM and Stierna P: The inflammatory response of the sinus and nasal mucosa during sinusitis: Implications for research and therapy. Acta Otolaryngol Suppl 515: 38-44, 1994

26. Bernstein JM: The molecular biology of nasal polyposis. Curr Allergy Asthma Rep 1: 262-267, 2001

27. Wiede A, Jagla W, Welte T, Köhnlein T, Busk H and Hoffmann W: Localization of TFF3, a new mucus-associated peptide of the human respiratory tract. Am J Respir Crit Care Med 159: 1330-1335, 1999.

28. Tomasetto $\mathrm{C}$ and Rio MC: Pleiotropic effects of Trefoil Factor 1 deficiency. Cell Mol Life Sci 62: 2916-2920, 2006.

29. Cui YH, Wang YY and Liu Z: Transdifferentiation of Clara cell $10-\mathrm{kDa}$ protein secreting cells in experimental allergic rhinitis. Am J Rhinol Allergy 25: 145-151, 2011.

30. Bossenmeyer-Pourié C, Kannan R, Ribieras S, Wendling C, Stoll I, Thim L, Tomasetto C and Rio MC: The trefoil factor 1 participates in gastrointestinal cell differentiation by delaying G1-S phase transition and reducing apoptosis. J Cell Biol 157: 761-770, 2002.

31. Perry JK, Kannan N, Grandison PM, Mitchell MD and Lobie PE: Are trefoil factors oncogenic? Trends Endocrinol Metab 19: 74-81, 2008. 